\title{
RESOURCE-CENTRIC BUSINESS CONTINUITY AND RESILIENCY PLANNING
}

\author{
Taro KANNO \\ Chie ISHID $A^{2}$ \\ Takayuki KANESAKA, Risa OKADA, Takashi KAWAZOE \\ Kazuaki TAE ${ }^{4}$ \\ Jun SATO \\ 1) The University of Tokyo, Japan \\ 2) Japan Red Cross College of Nursing, Japan \\ 3) Carepro Inc., Japan \\ 4) LE.O.VE Inc., Japan \\ 5) Tokyo Health Care University, Japan
}

\begin{abstract}
This paper presents a new framework for simple design and evaluation of business continuity and resilience plan (BCP) for medical institutions based on the categories of resources required for business continuity and the three phases of emergency response: preparedness, response, and recovery. Considering home-visit nursing stations as a use case study, this work also presents the manner in which the proposed framework can be used for the design and evaluation of BCPs.
\end{abstract}

Keywords: Business continuity and resilience plan (BCP), Resource-centric framework, Medical institutions

\section{INTRODUCTION}

Business continuity and resilience plan (BCP) entails methods that ensure the continuity of critical business processes as well as quick recovery during emergencies [1]. The importance of preparing workable and effective BCPs has been widely acknowledged in many business sectors. While the primary purpose of a $\mathrm{BCP}$ is to ensure the survival of the organization, in the particular case of medical institutions, the BCP is crucial for saving the lives of patients and reducing preventable mortalities in the event of a disaster, which leads to greater resilience of the community and society.

There are many guidelines and step-by-step guides for creating BCPs; however, it is not an easy task to create BCPs, particularly for mid- and small-sized companies such as medical institutions that do not have specialized personnel and adequate budget. In fact, the present survey on the preparation of BCPs at disaster base hospitals conducted by the Japanese Ministry of Internal Affairs and Communications (MIC) in 2018 [2] revealed that many hospitals often struggle with BCP preparation because of the lack of skills and know-how. This suggests that it is difficult for non-experts in the field to follow existing guidelines and manuals; thus, it is necessary to develop a simpler and easier BCP guidelines. Under these circumstances, we are

(C) 2019 Authors. This is an Open Access article distributed under the terms of the Creative Commons AttributionNonCommercial 4.0 International License (http://creativecommons.org/licenses/by-nc/4.0), permitting all non-commercial use, distribution, and reproduction in any medium, provided the original work is properly cited.

ISBN: 978-91-88898-41-8

DOI: https://doi.org/10.15626/rea8.21 
now attempting to develop a simple framework of BCP focusing on resources for the three phases of emergency response: preparedness, response, and recovery.

In the next section, we briefly review the current status and problems of $\mathrm{BCP}$ creation for medical institutions in Japan. We also briefly describe the standard procedures to create BCPs based on ISO220301 and identify its problems and difficulties for successful application. Then, in Section 3, we present our proposed design framework based on the resources required for business continuity and how this framework can be used for BCP preparation and evaluation, followed by the conclusions and future work in Section 4 .

\section{CURRENT STATUS AND PROBLEMS IN BCP FOR MEDICAL INSTITUTIONS}

Following the Great Hanshin-Awaji Earthquake of 1995, the Japanese Ministry of Health, Labor and Welfare (MHLW) decreed that each prefectural government designate disaster-base hospitals (DBHs) to fulfil the requirement for a central role in providing medical services in local areas in the event of a disaster. In 2017, in response to another earthquake, the Kumamoto Earthquake, the MHLW revised the requirements for these DBHs and required them to formulate BCPs and to conduct disaster trainings based on them [3]. While the MHLW provided a preliminary guide for disaster response planning for medical institutions based on the concept of BCP [4] and conducted seminars for BCP preparation many times, the survey by the MIC in 2018 [2] revealed that the staff in $77.1 \%$ of the hospitals still found it difficult to prepare BCPs because they do not have staff with necessary skills and know-how. The survey also revealed that people in $68.6 \%$ of the hospitals were at a loss about where to start on preparing the BCPs owing to the lack of available knowledge and instructive information. One of the problems with the MHLW's guide is that there are no clear or sufficient explanations for the basic concept of a BCP, and even though it attempted to explain the difference between BCPs and response plans and manuals, the rules are still unclear and confusing. Another problem with the MHLW guide is that it only provides a table of contents, items to be decided, and a checklist for BCP evaluation, but does not explain how to create such content.

The Cabinet Office, Government of Japan, Small and Medium Enterprise Agency, and other public agencies have also provided guidelines for BCP creation for ordinary companies [6]. These guidelines provide step-by-step procedures to create BCPs based on the requirements of ISO220301; however, there is also a drawback to this. The next subsection reviews the ISObased standard procedures for BCP preparation and specifies its limitations and problems, in particular, for medical institutions.

\subsection{ISO-based BCP guideline and its problems}

ISO220301 specifies the general requirements for business continuity plan and management. Most of the guidelines [5-8] for BCP preparation and evaluation reflect the policy and requirements. The standard procedures for creating ISO-based BCPs are as follows:

1) Setting BCP policy and objectives: The common objectives for the BCP is that it should sustain critical business operations and recovery from disruptions. In this step, a BCP creator sets a priority for their business as well as the objectives for recovery time and level.

2) Performing risk assessment: To define the scope and assumptions for BCP, by listing potential threats and types of emergencies and estimating their probability of occurrence and impact on business operations.

3) Performing business impact analysis: In this step, the BCP creator estimates the loss and costs incurred by potential threats and business interruption. 
4) Developing recovery strategies and action plans: The fundamental strategy for implementation of BCPs is minimizing loss and recovery time.

For experts on managerial studies or safety engineering, the above procedures are understandable on a surface level because the methods used in each step, such as risk assessment and task analysis, are familiar. However, it still seems to be difficult when put into practice. One of the difficulties is that each method entails fundamental limitations. For example, while it is difficult to estimate the probability of occurrence of events precisely even in risk assessment of artifacts [9], it is more difficult and virtually impossible to estimate the occurrence probability of rare events and situations, which are the main concerns of BCPs. Another example is that in risk assessment and business impact analysis, we need to list tasks or business operations not only for normal situations but also emergencies; however, this is not easy because a task is an epistemic construct and difficult to identify exclusively and exhaustively in a concrete and consistent manner. The task lists and categories created in different hospitals are not necessarily the same, and it seems difficult to share this knowledge and evaluate the $\mathrm{BCP}$ via cross-reference. There are also difficulties underlying the procedure. The procedure is not actually a step-by-step process, but rather an iterative method, or the procedure is just a list of the things to decide that are interdependent; thus, there is no clear starting point.

For BCP creators without great skill or knowledge, such as those in medical institutions, these problems become nothing but a source of confusion. A framework or guideline for BCP that simpler and easier to use is therefore highly required.

\section{RESOURCE-CENTRIC BCP FRAMEWORK}

\subsection{Redefining emergency response and $\mathrm{BCP}$ from the viewpoint of resources}

To solve the problems and limitations in existing BCP guidelines, we are developing a framework for BCPs focusing on resources used for tasks and operations for business continuity and emergency response. The reason why we stress resources is that resources such as devices and materials are mostly ontological constructs; therefore, they are easier to list and discuss. There are some epistemic resources that are non-tangible, such as knowledge and skills or authority; however, these can be grounded and referable to persons who possess them. In addition, almost all the troubles and problems for business continuity under emergency circumstances stem from, in essence, the lack of resources such as loss caused by disaster damage and excessive demand during a period of scarcity.

Based on this resource-centric viewpoint, we redefined the emergency response as the efficient and effective allocation of resources during a shortage scenario in business and emergency response operations; the $\mathrm{BCP}$ is involves securing, managing, and recovering and procuring resources (sourcing) for business continuity. A conceptual formulation of the resource-centric $\mathrm{BCP}$ is shown in (1) below. Securing, managing, and sourcing correspond to the typical three phases of disaster prevention, that is, preparedness, response, and recovery.

$$
\mathrm{BCP}=\text { Resources } \times(\text { Securing }+ \text { Managing }+ \text { Sourcing })
$$

\subsection{Four basic types of resources}

A BCP guide published by the Small and Medium Enterprise Agency provides a checklist for BCP evaluation, in which five items are listed for assessment: human resources, physical

\footnotetext{
$8^{\text {th }}$ REA Symposium Embracing Resilience: Scaling up and Speeding up Kalmar, Sweden, June 24-27, 2019
} 
resources, monetary resources, information, and emergency response systems [6]. The first four items are resources that are of the common category, "Mono (objects), Hito (human), Kane (money), Jyouhou (information)", which is usually referred to as management resources or firm resources in business science in Japan. We use this familiar resource category and develop further details for BCP preparation. The next subsection provides a detailed category of each type of resource, taking BCPs for home-visit nursing stations as an example.

\subsection{Resource category for home-visit nursing stations}

Through discussion with co-authors working for home-visit nursing stations, we have refined this resource category as shown in Table 1. We included customers as human resource because the service cannot be provided without customers. These lists are still not comprehensive; however, they are relatively easy to create because most of these resources have written lists, such as a staff list, customer list, and purchase list. Once these resources for business continuity and recovery are listed, the next step considers how to secure, manage, and source them, which becomes the main objective of the BCP. Because the target objects are clear and concrete, requiring only the actions for these objects, we can expect that this presents a clear and nonconfusing guide even for staff without specialized knowledge and skills on BCP preparation.

\begin{tabular}{lll} 
Resource type & Category & Examples \\
\cline { 2 - 3 } Human & In-house staff & managers, specialists, office clerks, etc. \\
\cline { 2 - 3 } & Outsourcing staff & $\begin{array}{l}\text { specialists, office clerks, volunteers, outside } \\
\text { supports, etc. }\end{array}$ \\
\cline { 2 - 3 } & Customer & current customers, potential customers, etc. \\
\cline { 2 - 3 } Physical & Device & buildings, rooms, spaces, etc. \\
\cline { 2 - 3 } & Material & for medical care, medicines, hygiene products, etc. \\
\cline { 2 - 3 } & For Mobility & cars, bicycles, etc. \\
\cline { 2 - 3 } Monel & gasoline, gas, etc. \\
\hline \multirow{2}{*}{ Monetary } & Saily commodity & food, water, bedclothes, etc. \\
\cline { 2 - 3 } & Response cost & salary, etc. \\
\hline \multirow{3}{*}{$\begin{array}{l}\text { Information } \\
\text { /Data }\end{array}$} & Contact list & in-house and outsourcing staffs, customers, business \\
& & contacts, public offices, etc. \\
\cline { 2 - 3 } & Customer info. & contract information, clinical record, etc. \\
\cline { 2 - 3 } & Inventory data & material, device, fuel, commodity, etc. \\
\cline { 2 - 3 } & Financial data & -
\end{tabular}

Table 1. Resource categories for home-visit nursing stations.

\subsection{Framework for BCPs}

Based on the conceptual formulation of BCP in Equation (1), we created a framework that provides an overview of the things to be considered for a BCP in the form of a table of four resource types and three phases of emergency response. Table 2 shows the framework, and its contents represent the $\mathrm{BCP}$ items for home-visit nursing stations listed thus far through the discussions in our research group. Basically, "securing resource" includes protection and damage mitigation, stockpiling, and alternate preparation of resources, "managing resource" includes prioritization and how to maximize the effectiveness of the limited resource usage, and "sourcing" includes obtaining the resources in shortage for business continuity and recovery. For example, in BCPs for securing staff, first we need to make a plan for securing our 
own safety by preparing concrete safety measures such as securing racks to a wall, preparing helmets, and providing instructions on how to save our lives. It is also important and necessary for the BCP to have an emergency contact system to confirm the safety of the staff and a practical emergency gathering system. While safety measures and first response actions are usually well planned in many institutions, the importance of managing and sourcing human resource seems to be less noticed and are not sufficiently prepared. For BCP creators in medical institutions, this framework can be expected to not only provide an overview and guide for $\mathrm{BCP}$ preparation but also become a checklist to assess the completeness of the $\mathrm{BCP}$.

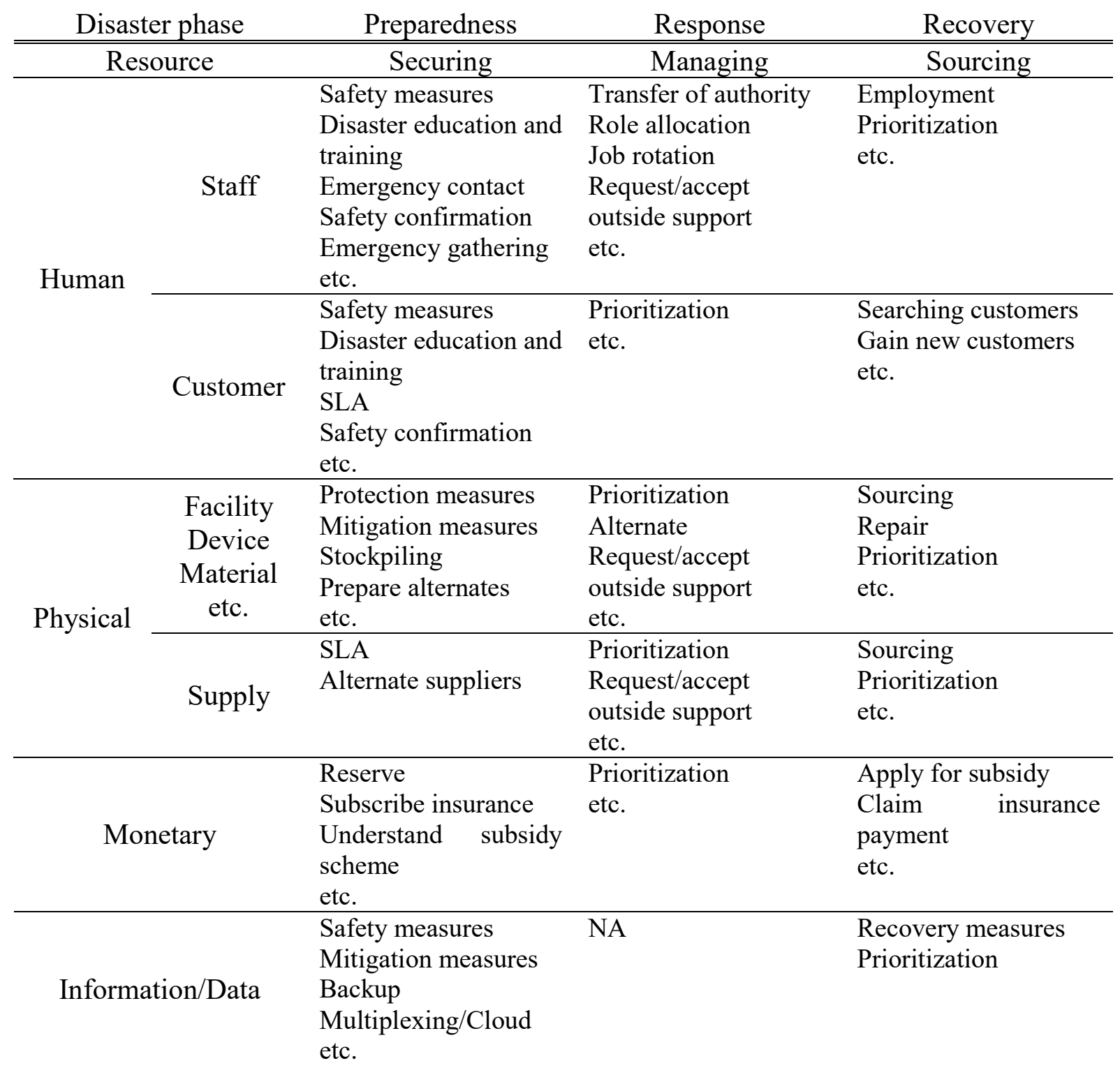

Table 2. Framework for a BCP guideline. 


\section{CONCLUSIONS AND FUTURE WORK}

This work first reviewed the problems and difficulties in BCP creation based on ISO220301; in particular, the problems for BCP creators without special skills and knowledge such as staff in medical institutions. Then, we proposed a conceptual formulation and simple framework for the $\mathrm{BCP}$ that reframes its contents as securing, managing, and sourcing resources for business continuity and recovery. This framework is still at the initial stage of development; however, the review response from several practioners was positive and garnered high expectations for further development of detailed BCP manuals and checklists.

The issues around BCP preparation that have not been considered currently and the research issues for the next step are listed as follows:

- Compliance with ISO220301 and ISO-based procedures: The ISO-based planning is a kind of top-down procedure in which business objective is firstly set followed by setting minimum business continuity objective (MBCO) and recovery time objective (RTO). This might make it difficult for non-experts to follow the procedures because without knowledge and information, it is generally impossible to set a practical objective. Our framework is rather more suited for a bottom-up approach in which understanding the current status of resource availability and business performance, followed by setting the objective accordingly. This is expected to make it easier for practioners to start planning. The next step is to develop detailed planning procedures which are based on our framework and fulfill the ISO requirements.

- Relationship between BCP and emergency response plan (ERP): The concept and target of BCPs and ERPs overlap to some extent. It is not practically necessary to distinguish them; however, it is good to provide some definition or hints to avoid unnecessary confusions. Based on the definition of emergency response and BCP in Section 3.1, the target of the $\mathrm{BCP}$ is to secure, manage, and source resources, and the ERP covers all other issues excluded from BCP that achives efficient and appropriate resource allocation in emergency, including efficient implementation of the BCP.

- Scope and assumptions: Our current discussions have focused on BCPs for natural disasters, in particular large earthquake, because it is a major and urgent concern in Japan . It is necessary to extend the framework to consider other emergency such as terrorist incidents and economic crisis.

\section{REFERENCES}

[1] ISO220301:2012, Societal security — Business continuity management systemsRequirements.

[2] Ministry of Internal Affairs and Communications, 2018, Survey results of the promotion of BCP preparation at disaster base hospitals (in Japanese):

http://www.soumu.go.jp/main_content/000580447.pdf (accessed 2019.4.12).

[3] Director General for Disaster Management, Cabinet Office, Government of Japan, 2015, Disaster Management in Japan (in Japanese):

http://www.bousai.go.jp/linfo/pdf/saigaipamphlet_je.pdf (accessed 2019.4.12).

[4] Ministry of Health, Labor, and Welfare, 2013, Guide for Disaster Response Planning for Medical Institutions Based on the Concept of BCP (in Japanese): https://www.mhlw.go.jp/file/06-Seisakujouhou-10800000-Iseikyoku/0000089048.pdf (accessed 2019.4.12). 
[5] Cabinet Office, Government of Japan, 2013, Guideline for Business Continuity Planning (in Japanese): http://www.bousai.go.jp/kyoiku/kigyou/pdf/guideline03.pdf (accessed 2019.4.12).

[6] Small and Medium Enterprise Agency, 2012, Towards the Promotion for BCP Implementation for Small and Medium Enterprises (in Japanese):

https://www.chusho.meti.go.jp/bcp/2012/download/24fyBCP.pdf (accessed 2019.4.12).

[7] Fulmer K.L., 2015, Business Continuity Planning $3^{\text {rd }}$ Ed., Rothstein Associates Inc.

[8] Sakata A., Kawamoto A., Ito N., Hatakeyama S., Isouchi C., and Shiraki W., 2013, A drawing approach of BCP for local government by the staff participation workshop, Journal of the Japan Society of Civil Engineering, SER F6, 69(2): I19-I24, https://doi.org/10.2208/jscejsp.69.I_19 (in Japanese with English abstract).

[9] Hollnagel E., 2012, FRAM: The Functional Resonance Analysis Method, CRC Press. 